\title{
Equations of Flying-Airboat Control Response Determination Movement Due to the Ground Effect
}

\author{
${ }^{1}$ H. Y. Akuma, ${ }^{2}$ M. Arifin \\ Faculty of Engineering and Life Sciences \\ Universiti Selangor, Bestari Jaya, Selangor, Malaysia
}

\begin{abstract}
A flying-airboat is a concept of a wing-in-ground (WIG) craft that seeks to take advantage of desirable characteristics within the ground effect, to provide versatile solutions to sea transportation needs in the Southeast Asia region. Previous research has shown that bodies traveling within the ground effect experience an improved lift-to-drag ratio, which translates to significantly better performance. The FAB is a concept that seeks to take advantage of these benefits through a design that facilitates motion entirely within the ground effect. By applying the relevant equations of motion and incorporating various physical design parameters of the concept, a non-linear model can be implemented on the MATLAB/Simulink platform. A simulation of the model shows relative improvements in the performance, response, and stability of the FAB concept subjected to the ground effect as compared to its motion within a free airstream.
\end{abstract}

Keywords: flying-airboat, wing-in-ground, ground effect, lift-to-drag ratio, performance, equations of motion.

\section{INTRODUCTION}

A flying-airboat $(\mathrm{FAB})$ is a new concept that incorporates the favorable attributes of motion within the ground effect into its design. The achievement of the concept includes a compound wing design as well as a tubular-skirt profile, similar to those in aircushion vehicles (ACVs) or hovercrafts. The wing design is idealized for the creation of lift with airflow over the airfoil section creating a dynamic air cushion [1]. On the other hand, the tubular skirt, in conjunction with moving air pumped from a set of fans, facilitates dynamic pressure that can potentially create an area of pressure differentiation underneath the body. An addition of a variable duct system that can vector the air within the skirt downwards has the potential to create lift along the Z-direction.

Determining the advantages of movement over ground effects and how physical design concepts respond to various control inputs are among the key factors in this study. Analysis of the framework of control over movement behavior in different situations can be made by developing a mathematical model of movement and applying it to the MATLAB platform. These results can further be used as a basis for changing and perfecting the design of the FAB. Therefore, the calculation of motion equations is applied to the design, which is first compiled by Mohd Zaid [2].

Previous studies in the marine and aviation fields have shown that a design combining the speed and efficiency of aircrafts with the practical implications of a marine-based craft provide a combined benefit of versatility. The design and development of the FAB concept thus seeks to provide a platform that can be used for fast and efficient sea-based missions involving search and rescue operations. To do so, Newtonian-Euler equations are modeled based on the physical parameters of the design and then tested on the MATLAB platform.

There are two forms of ground effect under consideration encompassing span-dominated ground effect (SDGE) and chorddominated ground effect (CDGE) [3]. Research conducted previously by Wetmore and Turner revealed that SDGE significantly reduces induced drag, whereas CDGE enhances a body's lift-producing capabilities [4]. The proposed design of the FAB concept seeks to utilize both these elements of the ground effect by encompassing a compound-wing design as well as a skirt facilitating dynamic pressure. However, this investigation focuses on the improvement of aerodynamic capabilities as a consequence of the compound wing design. To this effect, the motion of the FAB in a steady-state within the ground effect is tested against a model in unbounded free stream. The results of motion within the ground effect are then compared against motion of the FAB concept in the free airstream and conclusions deduced on the design implementation of the FAB concept. Renders of the tentative design are illustrated in Fig. 1. 

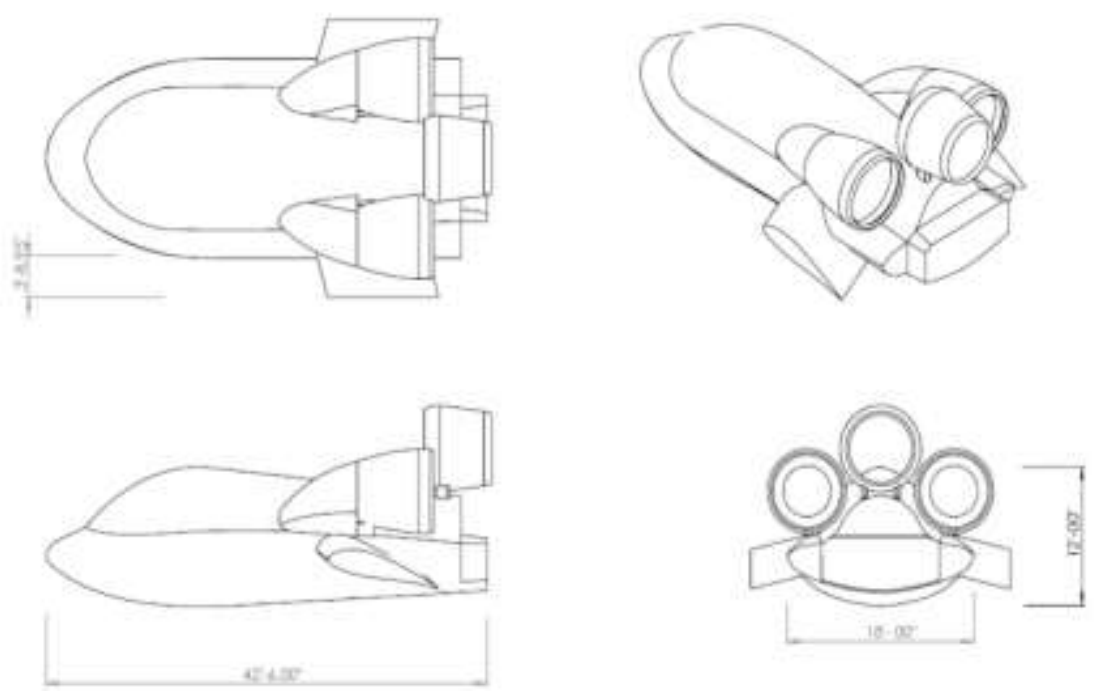

Fig. 1: Tentative design of the FAB Concept

\section{METHODOLOGY}

The methodology applied encompasses a series of steps which apply various relevant theories including aerodynamic theory, ground effect theory and control theory to deduce a numeric model that can be idealized on MATLAB. These steps are grounded in a non-linearized simulation framework encompassing the FAB's aerodynamics which is underpinned by twelve ordinary differential equations (ODEs) that define its state [5]. The model also incorporates external factors such as gravitational forces and wind disturbance. Fig. 2 below depicts the resultant dynamics and the form of their implementation on MATLAB.

The block diagram comprises of various components which can be defined as follows.

I. Aerodynamics - this block comprises of the relevant forces and moments affecting the FAB model as a result of aerodynamic capabilities. Parameters such wing span, the location of the center of gravity, angle of downwash and lift and drag coefficients thus play a determinate role within this block. It also affected by inputs that are pertinent to the control surface deflections .i.e. rudder, aileron and elevators.

II. Propulsion - this block comprises the forces and moments that are generated by the engine and contributing towards the overall motion of the body. Its inputs are thus synonymous with the throttle settings.

III. Gravity - The external independent forces acting on the body as a result of gravity are defined in this block.

IV. Wind corrections - This block represents the forces that act on the body due to atmospheric disturbances.

V. Atmosphere - Variables pertaining to air data that are obtained from onboard sensors are defined within this block. They include the Mach number, the Airspeed, Reynolds number and so on [5].

U denotes inputs which constitute the control vector $\overline{\mathbf{u}}$, whereas the states are defined by $\overline{\mathbf{X}}$. The definitions for the control and state vectors are also summarized in Tables $\mathbf{1}$ and $\mathbf{2}$.

Table 1: Control Input Definitions

\begin{tabular}{c|l|l|l|l}
\hline & Alphanumeric & Symbol & Name & Unit \\
\hline \multirow{5}{*}{$\overline{\mathbf{U}}=$} & $\delta_{\mathrm{A}}$ & Longitudinal deflection & $\mathrm{rad}$ \\
\cline { 2 - 5 } & $\mathrm{u}(1)$ & $\delta_{\mathrm{E}}$ & Lateral deflection & $\mathrm{rad}$ \\
\cline { 2 - 5 } & $\mathrm{u}(2)$ & $\delta_{\mathrm{R}}$ & Directional deflection & $\mathrm{rad}$ \\
\cline { 2 - 5 } & $\mathrm{u}(3)$ & $\delta_{\mathrm{TH} 1}$ & Throttle position 1 & - \\
\cline { 2 - 5 } & $\mathrm{u}(4)$ & $\delta_{\mathrm{TH} 2}$ & Throttle position 2 & - \\
\cline { 2 - 5 } & $\mathrm{u}(5)$ & $\mathrm{W}_{\mathrm{xE}}$ & Longitudinal wind & $\mathrm{m} / \mathrm{s}$ \\
\cline { 2 - 5 } & $\mathrm{u}(6)$ & $\mathrm{W}_{\mathrm{yE}}$ & Lateral wind & $\mathrm{m} / \mathrm{s}$ \\
\cline { 2 - 5 } & $\mathrm{u}(7)$ & $\mathrm{W}_{\mathrm{zE}}$ & Vertical wind & $\mathrm{s}$ \\
\cline { 2 - 5 } & $\mathrm{u}(8)$ & &
\end{tabular}

Table 2: State definitions

\begin{tabular}{c|l|l|l|l}
\hline & Alphanumeric & Symbol & Name & Unit \\
\hline \multirow{4}{*}{$\bar{x}(1)$} & $\mathrm{u}$ & Velocity in $\mathrm{x}$-axis & $\mathrm{m} / \mathrm{s}$ \\
\cline { 2 - 5 } & $\mathrm{x}(2)$ & $\mathrm{v}$ & Velocity in y-axis & $\mathrm{m} / \mathrm{s}$ \\
\cline { 2 - 5 } & $\mathrm{x}(3)$ & $\mathrm{w}$ & Velocity in z-axis & $\mathrm{n} / \mathrm{s}$ \\
\cline { 2 - 5 } & $\mathrm{x}(4)$ & $\mathrm{p}$ & Rate of roll & $\mathrm{rad} / \mathrm{s}$ \\
\cline { 2 - 5 } & $\mathrm{x}(5)$ & $\mathrm{q}$ & Rate of pitch & $\mathrm{rad} / \mathrm{s}$ \\
\cline { 2 - 5 } & $\mathrm{x}(6)$ & $\mathrm{r}$ & Rate of yaw & $\mathrm{rad} / \mathrm{s}$ \\
\cline { 2 - 5 }
\end{tabular}




\begin{tabular}{|l|l|l|l}
\hline $\mathrm{x}(7)$ & $\varphi$ & Roll angle & $\mathrm{rad}$ \\
\hline $\mathrm{x}(8)$ & $\theta$ & Pitch angle & $\mathrm{rad}$ \\
\hline $\mathrm{x}((9)$ & $\psi$ & Yaw/heading angle & $\mathrm{rad}$ \\
\hline $\mathrm{x}(10)$ & $\mathrm{x}$ & $\mathrm{x}$ position of CoG & $\mathrm{m}$ \\
\hline $\mathrm{x}(11)$ & $\mathrm{y}$ & $\mathrm{y}$ position of CoG & $\mathrm{m}$ \\
\hline $\mathrm{x}(12)$ & $\mathrm{z}$ & Z position of CoG & $\mathrm{m}$ \\
\hline
\end{tabular}

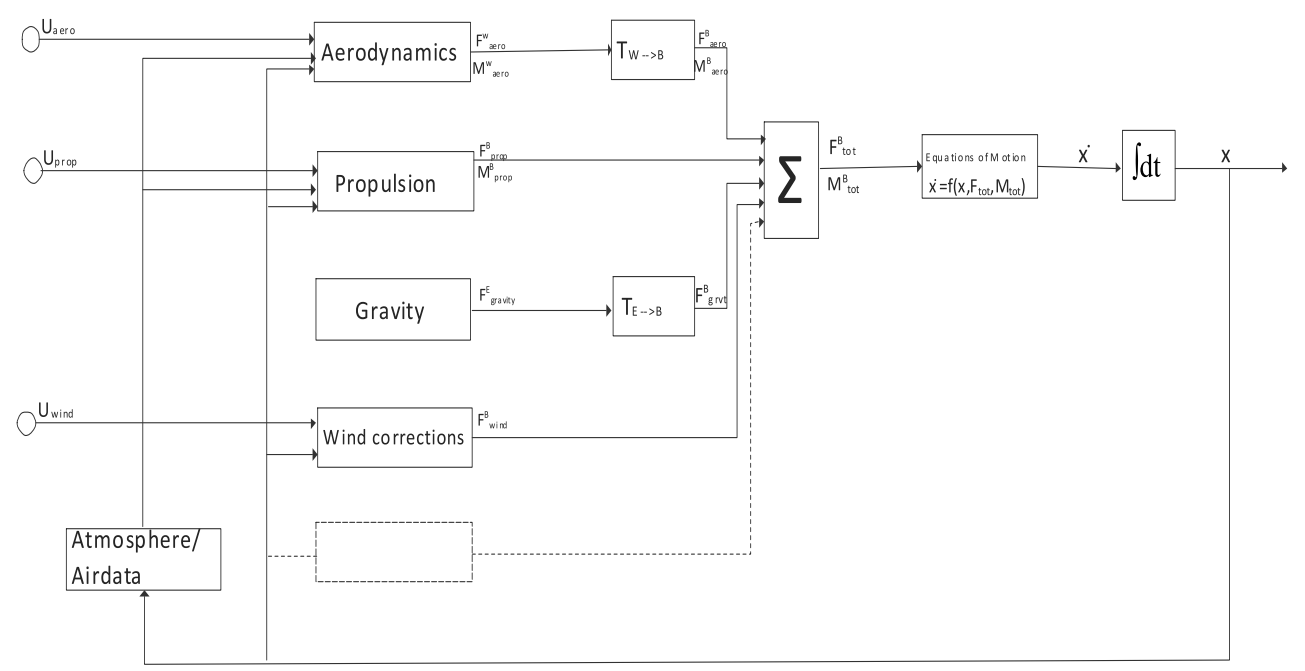

Fig. 2: Body dynamics block diagram [3]

The above definitions comprise the relevant design function and are applied within the MATLAB framework in the form of differential equations to give an overall. This deduction is shown in Fig. 3 below.

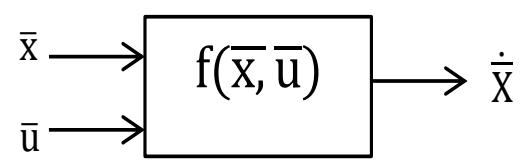

Fig. 3: Function combining the control inputs and the states

Consequently, the implementation of the model on the MATLAB entails a number of steps which are depicted in the following sections.

\section{Control Input Saturation}

A definition of the limits for the control inputs is established as the first step. This definition encompasses values beyond which the control inputs will have no effect on the physical motion of the model and are summarized in Eq. (1).

$$
\bar{u}=\left[\begin{array}{c}
\delta_{\text {aileron }} \\
\delta_{\text {tail }} \\
\delta_{\text {rudder }} \\
\delta_{\text {Thrust } 1} \\
\delta_{\text {Thrust } 2} \\
W_{x E} \\
W_{y E} \\
W_{z E}
\end{array}\right]=\left[\begin{array}{c}
u 1 \\
u 2 \\
u 3 \\
u 4 \\
u 5 \\
u 6 \\
u 7 \\
u 8
\end{array}\right] \in\left[\begin{array}{c}
{\left[-20^{\circ}, 20^{\circ}\right]} \\
{\left[-15^{\circ}, 5^{\circ}\right]} \\
{\left[-25^{\circ}, 25^{\circ}\right]} \\
{[0.2,10]} \\
{[0.2,10]} \\
{[\text { constant }]} \\
{[\text { constant }]} \\
{[\text { constant }]}
\end{array}\right] * \frac{\pi}{180}=\left[\begin{array}{c}
{[-0.349,0.349]} \\
{[-0.262,0.087]} \\
{[-0.436,0.436]} \\
{[0,0.175]} \\
{[0,0.175]} \\
{[- \text { inf }, \text { inf }]} \\
{[- \text { inf }, \text { inf }]} \\
{[- \text { inf }, \text { inf }]}
\end{array}\right]
$$

\section{Intermediate Variables}

Intermediate variables provide a relationship between the control inputs and the non-differential equations to determine the state vector. They include various predetermined variables that affect motion such as the airspeed, the angle of attack, the sideslip angle and the dynamic pressure. The intermediate variables applied in this investigation are as shown in Table 4. 
Table 3: Intermediate variables and application

\begin{tabular}{l|c}
\hline APPLICATION & VARIABLES \\
\hline Airspeed & $V_{A}=\sqrt{U^{2}+V^{2}+W^{2}} \rightarrow \sqrt{\boldsymbol{x}_{\mathbf{1}}^{2}+\boldsymbol{x}_{\mathbf{2}}^{2}+\boldsymbol{x}_{\mathbf{3}}^{2}}$ \\
Attack angle & $\beta=\tan ^{-1} \frac{w}{u} \rightarrow \tan ^{-1} \frac{\boldsymbol{x}_{\mathbf{3}}}{\boldsymbol{x}_{\mathbf{1}}}$ \\
Sideslip angle & $\sin ^{-1} \frac{V}{V_{A}} \rightarrow \sin ^{-1} \frac{\boldsymbol{x}_{\mathbf{2}}}{\boldsymbol{V}_{\boldsymbol{A}}}$ \\
Dynamic pressure & $Q=\frac{1}{2} \rho V_{A}^{2}$ \\
Translational velocity & $\bar{V}_{b}=\left[\begin{array}{l}u \\
v \\
W\end{array}\right]=\left[\begin{array}{l}\boldsymbol{x}_{\mathbf{1}} \\
\boldsymbol{x}_{\mathbf{2}} \\
\boldsymbol{x}_{\mathbf{3}}\end{array}\right]$ \\
Angular velocity vector & $\bar{\omega}_{b}=\left[\begin{array}{l}p \\
q \\
r\end{array}\right]=\left[\begin{array}{l}\boldsymbol{x}_{\mathbf{4}} \\
\boldsymbol{x}_{\mathbf{5}} \\
\boldsymbol{x}_{\mathbf{6}}\end{array}\right]$ \\
\hline
\end{tabular}

\section{The Lift Coefficient}

Determining the lift coefficient encompasses an examination of the compound wing in the conceptual design and its lift properties, as well as the tail section if any. The wing used in this investigation is the Eppler E857 whose lift curve is illustrated in the Fig. 4. The lift curve presented shows a critical angle of approximately 17 degrees and presents -5 degrees as the angle of attack where the lift produced is zero. Equations for the coefficient of lift of the wing body can thus be defined by Eq. 2 [6].

$$
C_{l w b}=\left\{\begin{array}{c}
n\left(\alpha-\alpha_{l=0}\right) \text { if } \alpha \leq \frac{17 \pi}{180} \text { otherwise } \\
a_{3} \alpha^{3}+a_{2} \alpha^{2}+a_{1} \alpha+a_{0}
\end{array}\right.
$$

Whereby: $\alpha_{l=0}$ is the angle of attack where lift is 0

$\mathrm{n}=5.5$ is the slope of the lift curve

$\mathrm{a}_{3}=-768.5$

$\mathrm{a}_{2}=609.2$

$a_{1}=-155.2$

$\mathrm{a}_{0}=15.212$ 


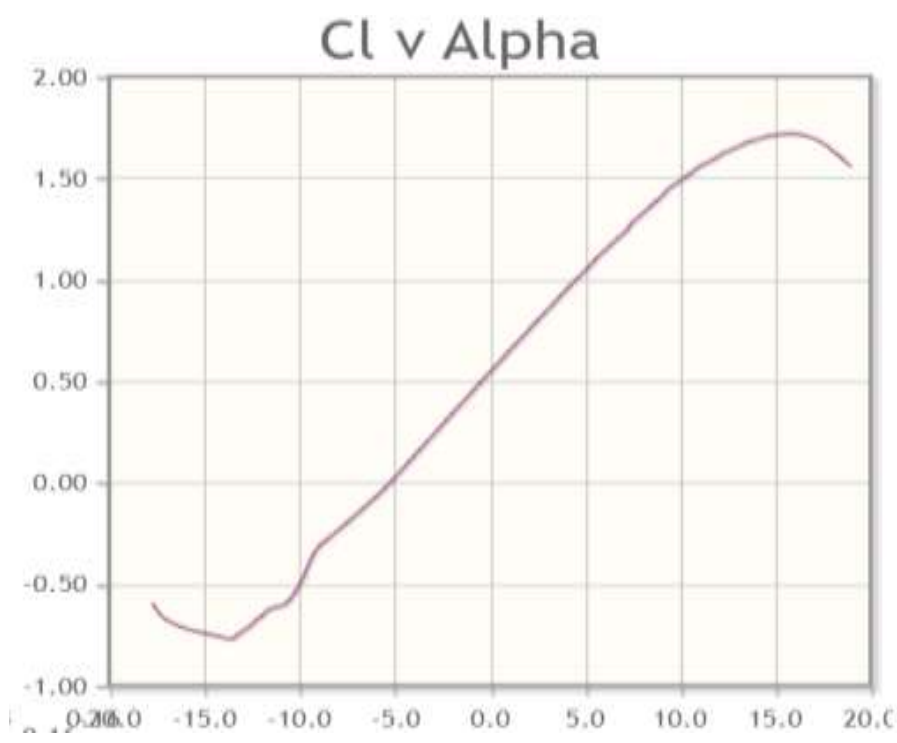

Figure 4. Lift Curve for Eppler E857 [7]

After initial tests, a small tail section was added to the model to increase its stability. The lift produced by this section is based on the angle of attack of the tail section which is defined by Eq. 3 [6].

$$
\alpha_{t}=\alpha-\varepsilon+u_{2}+1.3 x_{5} \frac{l_{t}}{V_{A}}
$$

Where: $\varepsilon($ downwash $)=\frac{\partial \varepsilon}{\partial \alpha}\left(\alpha-\alpha_{l=0}\right)$

$$
\mathrm{u}_{2}=\text { tail angle }
$$

$1.3 x_{5} \frac{l_{t}}{V_{A}}=$ dynamic pitch response

$l_{t}$ is the distance between the wing \& tail

Based on the angle of attack deduced above, the coefficient of lift of the tail section is given below [6].

Where: $\mathrm{S}_{\mathrm{t}}$ is the tail plan form area

$$
C_{l t}=3.1 \frac{S_{t}}{S} \alpha_{t}
$$

$\mathrm{S}$ is the wing plan form area

A combination of the wing body and tail coefficients gives the overall coefficient of lift as shown by Eq. 5 .

$$
C_{L}=C_{l w b}+C_{l t}
$$

The ground effect however, has an influence on the coefficient of lift and therefore, in the model simulated within the ground effect, an influence parameter is incorporated as follows.

$$
C_{L}^{\prime}=\frac{C_{L}}{\left(1-\sigma_{g e}\right)}
$$

Whereby: $\mathrm{C}_{\mathrm{L}}$ ' is the coefficient of lift within the ground effect

$\mathrm{C}_{\mathrm{L}}$ is the total coefficient of lift

$\sigma_{g e}$ is the ground effect influence parameter given by:

Whereby: $\mathrm{h}$ is the height and,

$$
\sigma_{g e}=e^{-2.48\left(\frac{2 h}{b}\right)^{0.768}}
$$

$\mathrm{b}$ is the wing span

\section{The Coefficient of Drag}

The drag coefficient is based upon Computer Fluid Dynamics (CFD) simulations done in previous research conducted by Mohd Zaid [2]. A predetermined value is thus employed.

\section{Sideforce Coefficent}

Forces acting along the y axis also contribute to the control response for the model and are based on predetermined constants. Because, the sideslip angle and rudder control input are assumed to be zero for the purpose of this research however, this force is assumed to be negligible. Nevertheless, the applicable equation is presented by Eq. 7 as follows.

Whereby: $\beta$ is the sideslip angle

$$
C_{Y}=-1.6 \beta+0.24 u_{3}
$$

$\mathrm{u}_{3}$ is the directional control (rudder) angle

\section{Stability Frame}

The resultant coefficients and forces are in the stability frame and presented by Eq. 8 and 9 as follows. 


$$
\begin{gathered}
\bar{C}_{F}^{\text {Stblty }}=\left[\begin{array}{l}
\text { Force along } x \text { axis } \\
\text { Force along } y \text { axis } \\
\text { Force along } z \text { axis }
\end{array}\right]=\left[\begin{array}{c}
-C_{D} \\
C_{Y} \\
-C_{L}
\end{array}\right] \\
\bar{F}_{A}{ }^{S}=\left[\begin{array}{c}
-D \\
Y \\
-L
\end{array}\right]^{S}=\left[\begin{array}{c}
-C_{D} \cdot Q \cdot S \\
C_{Y} \cdot Q \cdot S \\
-C_{L} \cdot Q \cdot S
\end{array}\right]
\end{gathered}
$$

The above forces in the stability frame are transformed to the body frame of reference as follows.

$$
\bar{F}_{A}^{B}=C_{b / s}(\alpha) * \bar{F}_{A}^{S}
$$

Whereby: $C_{b / s}(\alpha)$ is the transformation matrix about the angle of attack denoted as follows:

$$
C_{b / s}(\alpha)=\left[\begin{array}{ccc}
\cos \alpha & 0 & -\sin \alpha \\
0 & 1 & 0 \\
\sin \alpha & 0 & \cos \alpha
\end{array}\right]
$$

\section{Coefficients of Moment}

Moments of coefficients for the model are given about both the aerodynamic center of the body, and its center of gravity. These moments are based upon Newtonian laws of motion. Derivation for moment about the aerodynamic center is presented as follows [6].

$$
\bar{C}_{\text {M.ac }}=\left[\begin{array}{l}
C_{l . a c} \\
C_{m . a c} \\
C_{\text {n.ac }}
\end{array}\right]=\bar{n}+\frac{\partial C_{m}}{\partial x} \cdot \bar{\omega}_{b}+\frac{\partial C_{m}}{\partial u} \cdot\left[\begin{array}{l}
u_{1} \\
u_{2} \\
u_{3}
\end{array}\right]
$$

Whereby: $\bar{n}$ is a moment effect defining the static behavior and can be broken down as follows:

In the above equation:

$$
\bar{n}=\left[\begin{array}{c}
-1.4 \beta \\
-0.59-3.1 \frac{S_{t} l_{t}}{S \bar{c}}(\alpha-\varepsilon) \\
\left(1-\alpha \frac{180}{\pi}\right) \beta
\end{array}\right]
$$

$\overline{\mathrm{c}}$ is the mean aerodynamic chord length

$l_{t}$ is the distance between the aerodynamic center of the tail and the CoG

$$
\begin{gathered}
\frac{\partial C_{m}}{\partial x}=\left[\begin{array}{ccc}
-11 & 0 & 5 \\
0 & -4.03 \frac{S_{t} l_{t}^{2}}{S \bar{c}} & 0 \\
1.7 & 0 & -11.5
\end{array}\right] * \frac{\bar{c}}{V_{A}} \\
\frac{\partial C_{m}}{\partial u}=\left[\begin{array}{ccc}
-0.6 & 0 & 0.22 \\
0 & -3.1 \frac{S_{t} l_{t}}{S \bar{c}} & 0 \\
0 & 0 & -0.63
\end{array}\right]
\end{gathered}
$$

The terms $\frac{\partial C_{m}}{\partial x}$ and $\frac{\partial C_{m}}{\partial u}$ are used to define the effectiveness of the controls The moment about eh aerodynamic center is therefore as follows.

$$
\bar{M}_{A . a c}=\bar{C}_{M . a c} \cdot Q \cdot S \cdot \bar{c}
$$

On the other hand, the moment coefficient bout the center of gravity can be defined as follows.

Whereby:

$$
\bar{M}_{A . c g}=\bar{M}_{A . a c}+\bar{F}_{A}^{B} \cdot\left(\bar{r}_{c g}-\bar{r}_{a c}\right)
$$

$$
\bar{r}_{c g}=\left[\begin{array}{l}
X_{c g} \\
Y_{c g} \\
Z_{c g}
\end{array}\right]=\left[\begin{array}{l}
x \text { position of } C o G \\
y \text { position of } C o G \\
z \text { position of } C o G
\end{array}\right]=\left[\begin{array}{c}
0.25 \bar{c} \\
0 \\
-0.30 \bar{c}
\end{array}\right]
$$

and 


$$
\bar{r}_{a c}=\left[\begin{array}{l}
X_{a c} \\
Y_{a c} \\
Z_{a c}
\end{array}\right]=\left[\begin{array}{l}
x \text { position of aero center } \\
y \text { position of aero center } \\
z \text { position of aero center }
\end{array}\right]=\left[\begin{array}{c}
0.21 c \\
0 \\
0
\end{array}\right]
$$

\section{Propulsion Effects}

The forces and moments as a result of the engine thrust are also derived to come up with the dynamic model. Thrust is produced by a dual-fan setup and can be modeled as follows.

$$
F_{i}=\delta_{t h . i} \cdot m \cdot g
$$

Whereby: $F_{i}$ represents the thrust force produced by the ith engine(fan)

$\delta_{\text {th.i }}$ is the control input for the ith fan

$\mathrm{m}$ is the mass of the Airboat

$\mathrm{g}$ is the gravitational force

The representation above can be related to the control inputs for thrust and be denoted as follows.

$$
u_{4}, u_{5} \leq \frac{10 \pi}{180}\left\{\begin{array}{l}
F_{1}=U_{4} \cdot m \cdot g \\
F_{2}=U_{5} \cdot m \cdot g
\end{array}\right.
$$

In the body frame, the above forces as a result of propulsion are as follows.

$$
\begin{gathered}
\bar{F}_{E i}{ }^{b}=\left[\begin{array}{c}
F_{i} \\
0 \\
0
\end{array}\right]^{b} \\
\bar{F}_{E}{ }^{b}=\bar{F}_{E 1}{ }^{b}+\bar{F}_{E 2}{ }^{b}
\end{gathered}
$$

The above principle was also used to simulate propulsion as a result of the thrust force being vectored vertically, and parallel to but opposite the force of gravity. This was as a means to simulate the resultant forces produced by a tubing mechanism that is expected to be incorporated into the FAB's design, so as to facilitate VTOL. The resultant forces produced as a result of the nozzles underneath the FAB were modeled as follows.

$$
\begin{aligned}
& F_{3}=F_{1} \\
& F_{4}=F_{2}
\end{aligned}
$$

Where $\mathrm{F}_{3}$ and $\mathrm{F}_{4}$ are the vertical forces vectored to the right and left nozzle systems underneath the FAB

As representation of this forces in the body frame was subsequently determined as follows.

$$
\begin{gathered}
\bar{F}_{E i}{ }^{b}=\left[\begin{array}{c}
0 \\
0 \\
F_{i}
\end{array}\right]^{b} \\
\bar{F}_{E}{ }^{b}=\bar{F}_{E 3}{ }^{b}+\bar{F}_{E 4}{ }^{b}
\end{gathered}
$$

The vertical mode of the FAB is activated once the control inputs $\mathrm{u} 4$ and u5 are at a setting of $60 \%$ or above. This translates to about 0.12 radians out of a maximum setting of 0.17 (10 degrees). Since the two throttle inputs are operated simultaneously, these translates to a value of 0.24 , which is represented with the following MATLAB syntax.

$$
\begin{gathered}
\text { if }(\mathbf{u} 4+\mathbf{u}>0.24) \\
\text { FE_b = FE_bv; } \\
\text { elseif }(\mathbf{u} 4+\mathbf{4} 5<0.24) \\
\text { FE_b = FE_bh; } \\
\text { end }
\end{gathered}
$$

Where; FE_bv is the vertical force in the body frame,

FE_bh is the horizontal force in the body frame and

FE_b is the Total Engine Force.

The above syntax dictates that a throttle input above 0.12 radians for each engine will activate the vertical force prompting a velocity along the z-axis. On the other hand, a throttle input below 0.12 radians for each engine maintains horizontal thrust and velocity along the $\mathrm{x}$-axis. 
The moments produced as a result of the propulsion effects are also of relevance. The determination of these forces is based on the application points of the thrust in relation to the center of gravity, given in the primary axes. This derivation is as follows.

$$
M_{E c g . i}{ }^{b}=\bar{\mu}_{i}^{b} \cdot \bar{F}_{E i}^{b}
$$

Whereby: $\bar{\mu}_{i}^{b}$ is the moment arm and can be expressed as follows

$$
\bar{\mu}_{i}^{b}=\left[\begin{array}{l}
X_{c g}-X_{A P T . i} \\
Y_{A p T . i}-Y_{c g} \\
Z_{c g}-Z_{A P T . i}
\end{array}\right]
$$

The overall propulsion moment about the center of gravity along the $\mathrm{x}$-axis is therefore determined as follows.

$$
M_{E c g}{ }^{b}=M_{E c g .1}^{b}+M_{E c g .2}^{b}
$$

On the other hand, the concept has the potential to have a propulsion moment about the center of gravity but along the $\mathrm{z}$-axis. This is represented as follows.

$$
M_{E c g}^{b}=M_{E c g .3}^{b}+M_{E c g .4}^{b}
$$

In the above equation the equated components represent resultant engine moments as a result of the nozzles found in the skirt. Similar to the forces, these moments are activated by a throttle setting above 0.12 radians.

Gravity Effect

Forces acting on the body as a result of gravity are also defined as part of the equations of motion. This is done as follows.

$$
\bar{F}_{g}=m \cdot \bar{g} \quad \bar{F}_{g}{ }^{e}=\left[\begin{array}{l}
0 \\
0 \\
g
\end{array}\right] \cdot m
$$

The forces are transformed from the earth frame to the body frame as follows [6].

$$
\bar{F}_{g}^{b}=C_{b / e}(\varphi, \theta, \psi) \bar{F}_{g}^{e}
$$

The result of the above transformation is as follows.

$$
\bar{F}_{g}{ }^{b}=\left[\begin{array}{c}
-g \sin x 8 \\
g \cos x 8 \sin x 7 \\
g \cos x 8 \cos x 7
\end{array}\right] \cdot m
$$

Consequently, the derived forces and moments are implemented on the MATLAB platform using tools such as m-text and a model with the interpreted MATLAB function on Simulink. The results are presented in the following section.

\section{RESULTS}

Implementation of the equations derived above is done in conjunction with various constant values, which define desirable control inputs and initial states. The results based on simulations run through MATLAB computational software are presented in the following sections.

\section{Control Inputs}

For the purpose of this investigation, the control input values chosen include elevator deflection set at $-0.087 \mathrm{rad}\left(\approx 5^{\circ}\right)$ and a throttle setting of $0.14 \mathrm{rad}\left(\left(\approx 8^{\circ}\right)\right.$. All the other control inputs are assumed to have a constant value of zero. These inputs are simulated as constants for a period of 180 seconds and their results plotted in Fig. 5.

\section{State Simulation}

On the other hand, inputs chosen for the initial state include a longitudinal speed (along X-axis) of $50 \mathrm{~m} / \mathrm{s} \quad(\approx 58 \mathrm{knots})$ and a height of 1.5 meters. The pitch angle also corresponds to the elevator deflection at 0.087 rads. These inputs are demonstrated as follows.

$$
\mathrm{x} 0=[50 ; 0 ; 0 ; 0 ; 0 ; 0 ; 0 ; 0.087 ; 0 ; 0 ; 0 ;-1.5]
$$

The control inputs above are simulated on the MATLAB platform for a period of 180 seconds and are plotted for both a model within ground effect and one where the ground effect parameter is not considered. Fig. 6 represents a model with the ground effect parameter whereas Fig. 7 is for a model lacking ground effect influence. 


\section{Angle of Attack}

To demonstrate the effect of the angle of attack has on the stability of the model, simulations are also done at optimal $30 \mathrm{~m} / \mathrm{s}$ longitudinal velocity and at various angles of attack including at 5, 17 and 30 degrees. It should be noted that the critical angle of attack is 17 degrees and that the input of the states is in form of radians. The corresponding pitch angles are thus 0.087 rad, 0.297 rad and 0.525 rad. The results are presented in Fig. 8, Fig. 9 and Fig. 10.

\section{Vertical Force}

A vertical mode was also simulated to depict how vectored thrust forces from the engines to nozzles underneath the Airboat model would affect its dynamic capabilities. The vertical mode was programmed to be initiated with a throttle setting of above $66 \%$ on each engine translating to a setting above 0.12 rads for control inputs U4 and U5. Fig. 11 shows the plotted results when the model was in horizontal mode $\left(0.11\right.$ rads for $\left.\mathbf{u}_{4}, \mathrm{u}_{5}\right)$, whereas Fig. 12 shows a simulation of the vertical mode at a throttle setting of 0.13 rads.

\begin{tabular}{|c|c|c|c|c|c|c|c|c|c|}
\hline 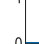 & 1 & & | & 1 & $\mid$ & & 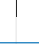 & T & $-u_{1}$ \\
\hline . & 1 & 1 & 1 & 1 & | & l & 1 & 1 & \\
\hline 0 & 20 & 40 & 60 & 80 & 100 & 120 & 140 & 160 & 180 \\
\hline 0.5 & 1 & 1 & 1 & 1 & 1 & 1 & 1 & 1 & $-u_{2}$ \\
\hline $\begin{array}{r}-0.5 \\
-1\end{array}$ & 1 & 1 & 1 & 1 & 1 & 1 & 1 & 1 & - \\
\hline 0 & 20 & 40 & 60 & 80 & 100 & 120 & 140 & 160 & 180 \\
\hline 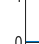 & 1 & 1 & 1 & 1 & 1 & 1 & 1 & 1 & $-u_{3}$ \\
\hline . & $\perp$ & 1 & 1 & 1 & 1 & 1 & 1 & 1 & \\
\hline 0 & 20 & 40 & 60 & 80 & 100 & 120 & 140 & 160 & 180 \\
\hline 0.5 & I & 1 & $T$ & 1 & 1 & 1 & 1 & 1 & $-u_{4}$ \\
\hline-0.5 & 1 & 1 & 1 & 1 & 1 & 1 & 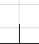 & 1 & - \\
\hline 0 & 20 & 40 & 60 & 80 & 100 & 120 & 140 & 160 & 180 \\
\hline $\begin{array}{r}1 \\
0.5\end{array}$ & $T$ & 1 & 1 & 1 & 1 & 1 & 1 & 1 & $u_{5}$ \\
\hline-0.5 & 1 & 1 & 1 & 1 & 1 & 1 & 1 & 1 & - \\
\hline 0 & 20 & 40 & 60 & 80 & 100 & 120 & 140 & 160 & 180 \\
\hline 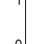 & 1 & 1 & 1 & 1 & 1 & 1 & 1 & 1 & $-u_{6}$ \\
\hline -1 & 1 & 1 & 1 & 1 & 1 & 1 & 1 & 1 & \\
\hline 0 & 20 & 40 & 60 & 80 & 100 & 120 & 140 & 160 & 180 \\
\hline 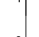 & 1 & 1 & 1 & 1 & 1 & 1 & 1 & 1 & $-u_{7}$ \\
\hline L & 1 & 1 & 1 & 1 & 1 & 1 & 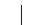 & 1 & \\
\hline 0 & 20 & 40 & 60 & 80 & 100 & 120 & 140 & 160 & 180 \\
\hline 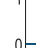 & 1 & 1 & 1 & 1 & 1 & 1 & 1 & 1 & $\longrightarrow u_{8}$ \\
\hline 1 & 1 & $\perp$ & $\perp$ & 1 & 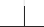 & 1 & 1 & 1 & \\
\hline 0 & 20 & 40 & 60 & 80 & 100 & 120 & 140 & 160 & 180 \\
\hline
\end{tabular}

Fig. 5: Graph showing Control Input Plots over a period of 180 seconds 

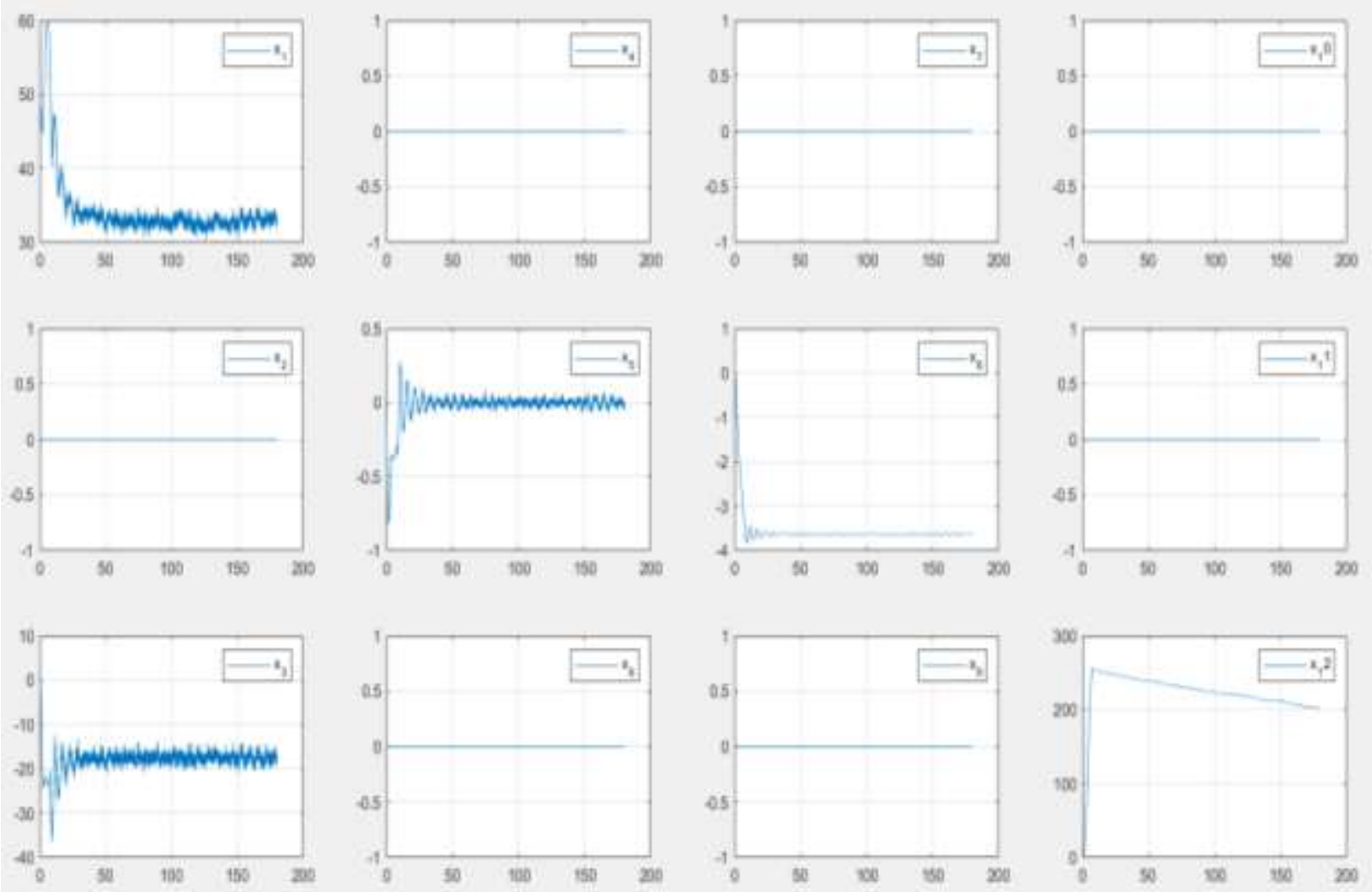

Fig. 6: Output of the States with the Ground Effect Influence
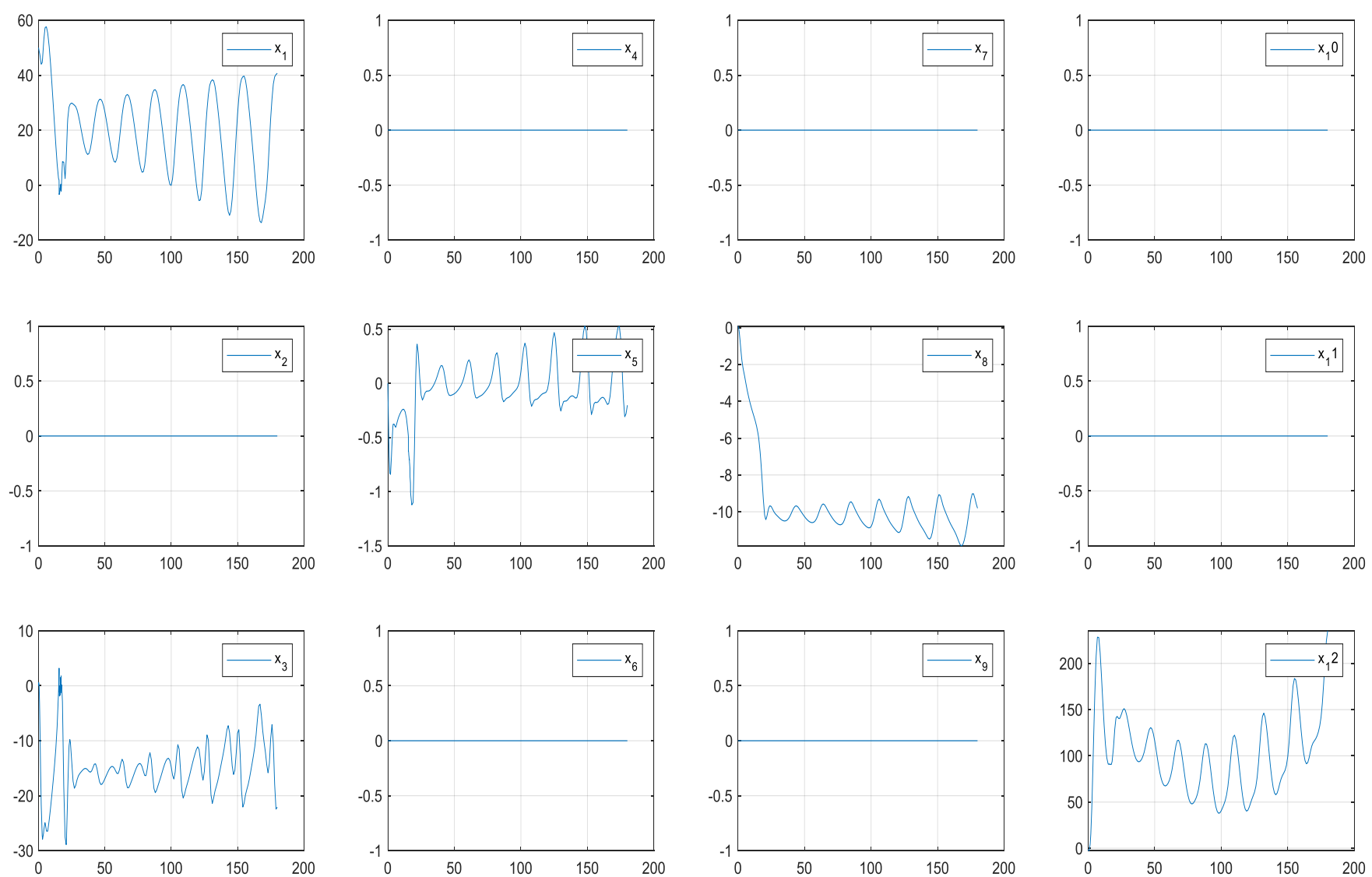

Fig. 6: Output of the States without the Ground Effect Influence 

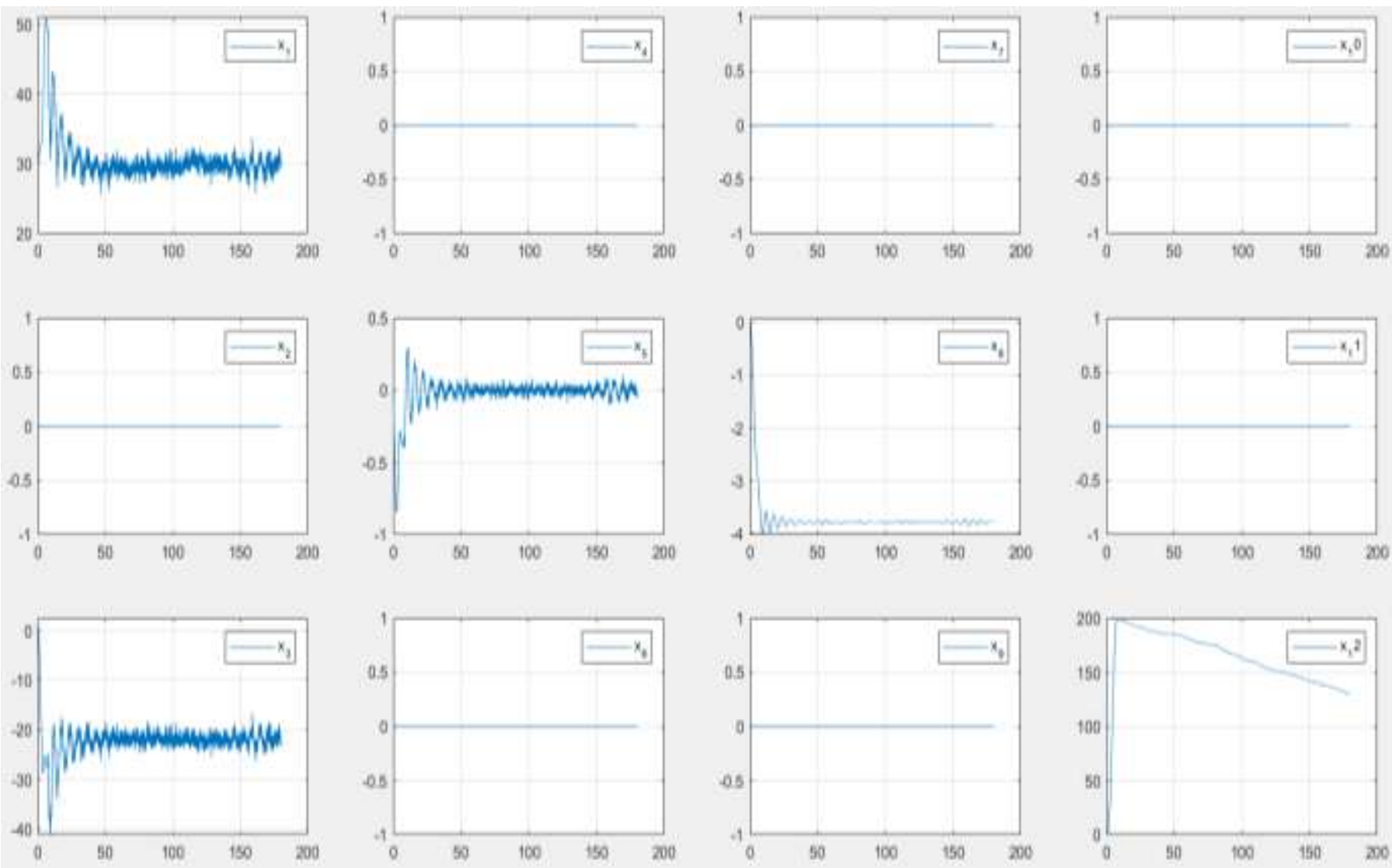

Fig. 7: Output of the States with Ground Effect Influence at $5^{\circ}$ Pitch Angle
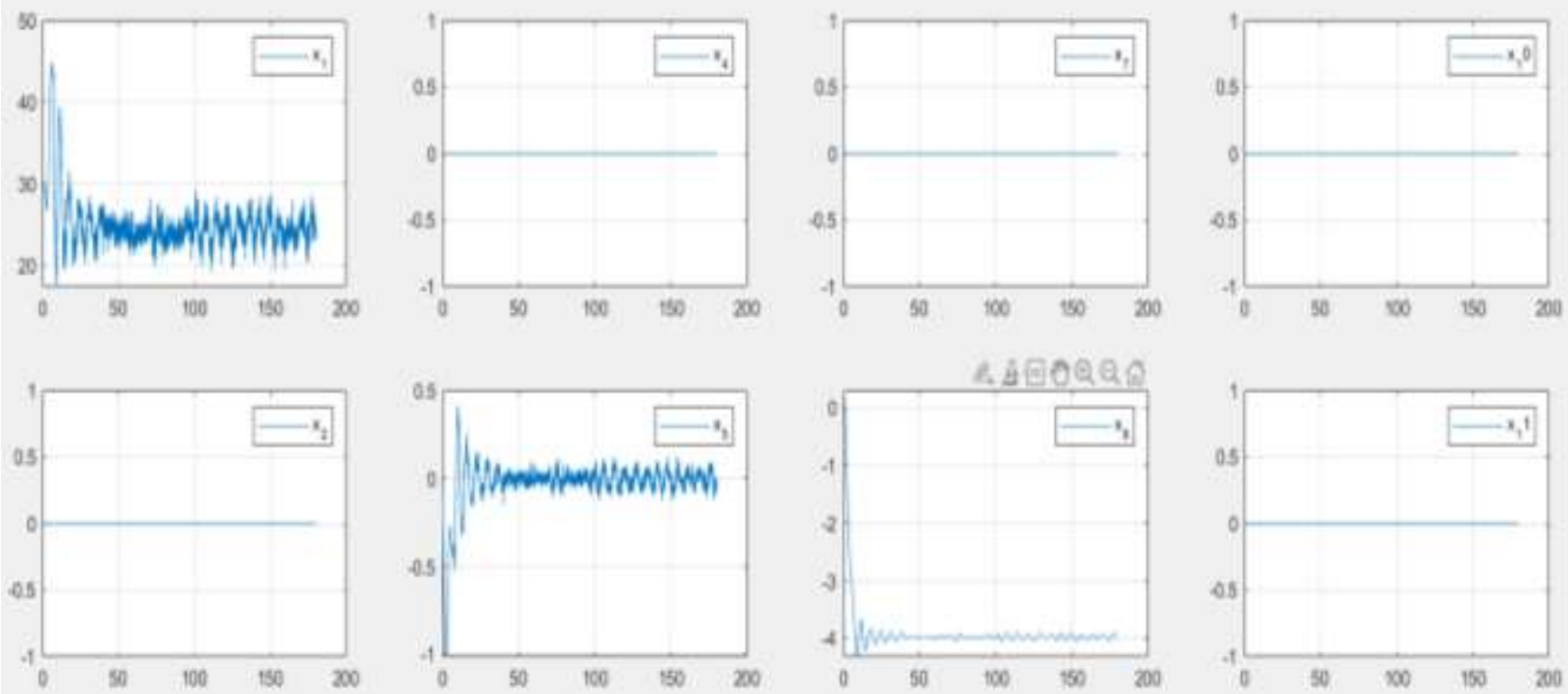

4. $\mathrm{B} 0 \mathrm{QQQ} \mathrm{Q}$
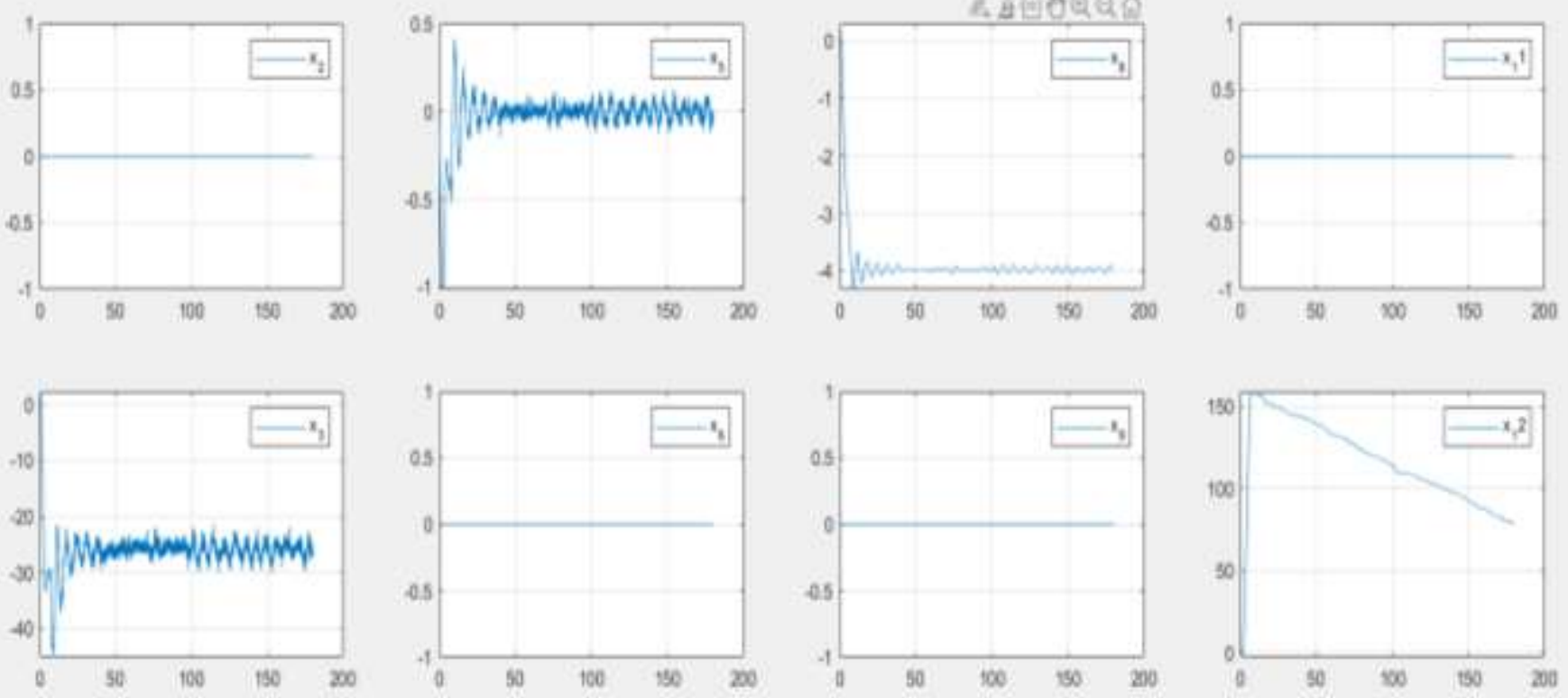

Fig. 8: Output of the States with Ground Effect Influence at $17^{\circ}$ Pitch Angle 

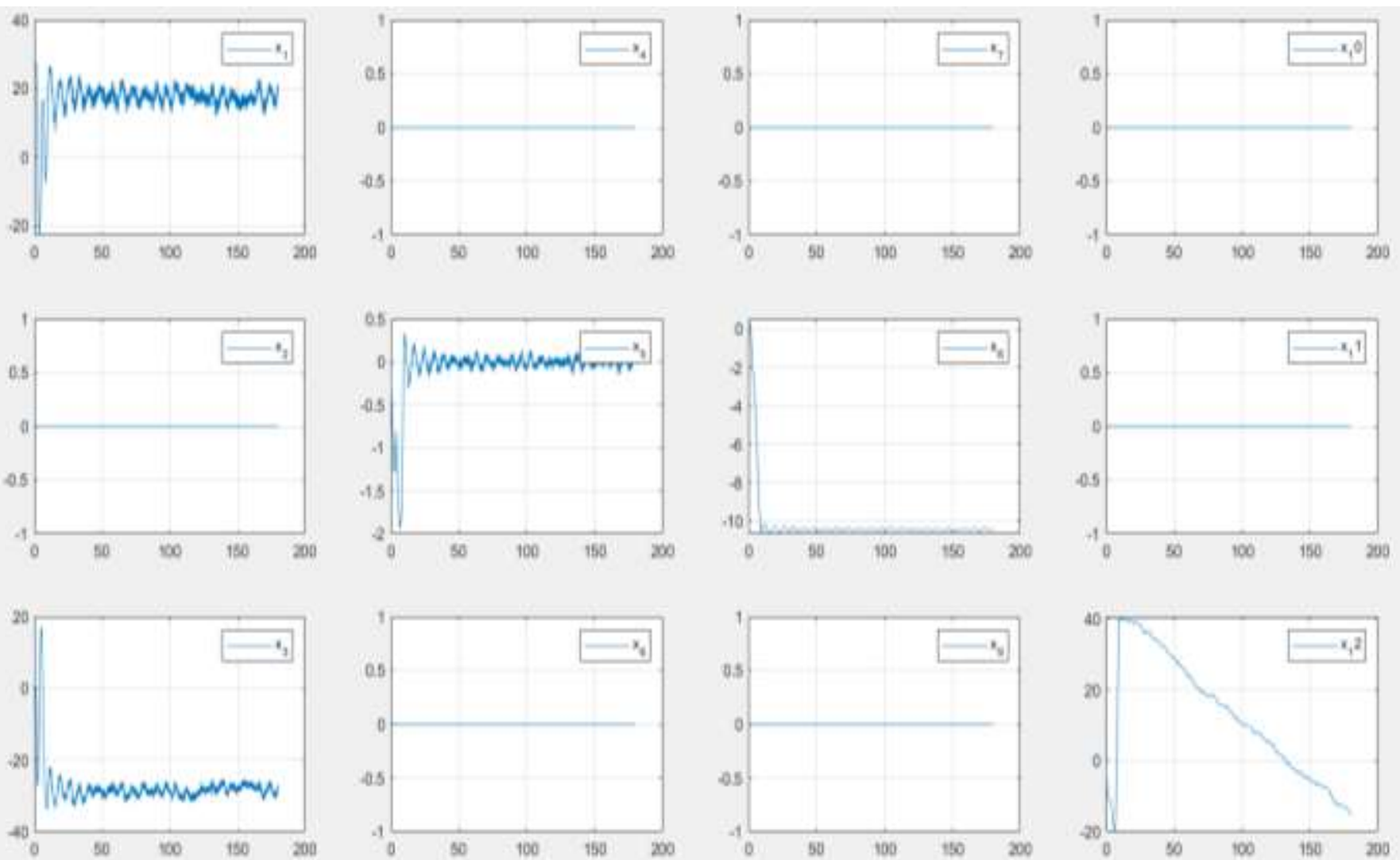

Fig. 9: Output of the States with Ground Effect Influence at $30^{\circ}$ Pitch Angle
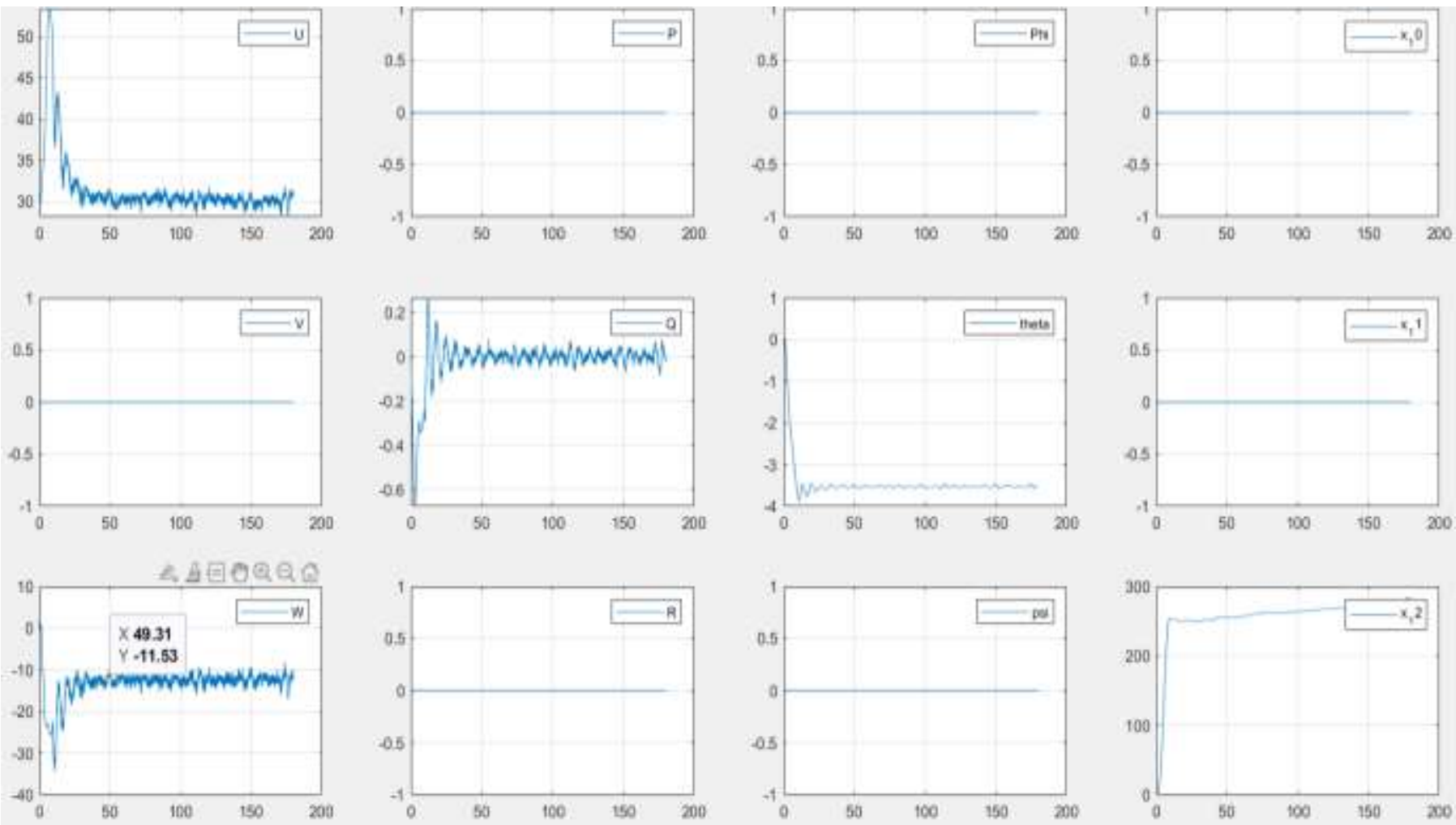

Fig. 10: Output of the States in horizontal motion at 63\% Throttle Setting 

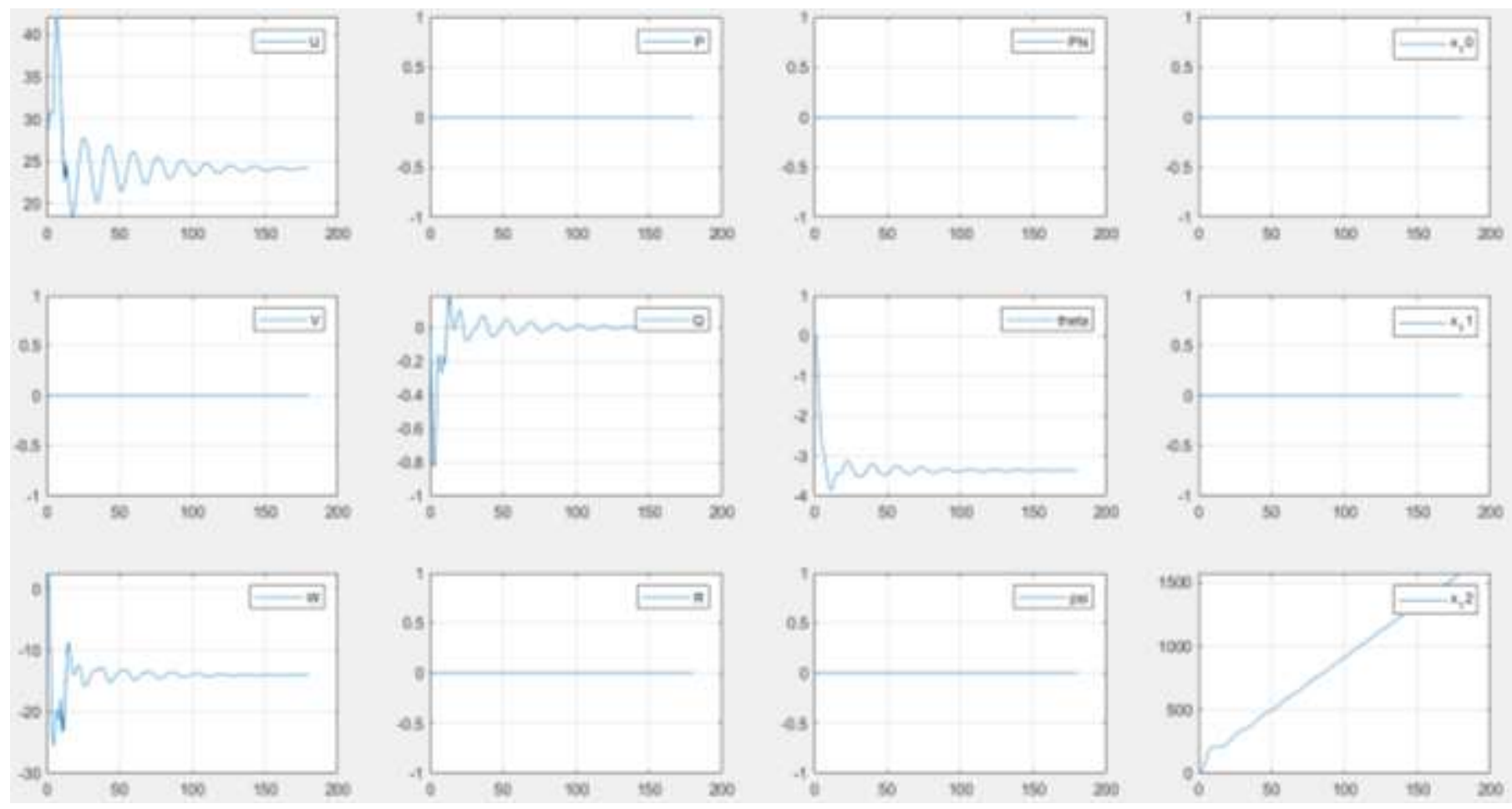

Fig. 11: Output of the States when vertical mode is activated at $74 \%$ Throttle Setting

\section{DISCUSSION}

\section{Velocity in $\mathrm{X}$-axis}

In the results presented above, the examination of velocity in the $\mathrm{x}$-direction provides a contrast between a model within the ground effect and one moving in free air stream, which is illustrated Fig. 13. In the presented plots, the graph on the right shows simulation within ground effect whereas the graph on the left shows simulation without ground effect. Based on the plots, it is evident that the model exhibits superior stability characteristics within the ground effect, as compared to when in motion within an unbound airstream. This is synonymous with a waveform of relatively diminished amplitudes and higher frequency. After an initial application of a $50 \mathrm{~m} / \mathrm{s}$ velocity, the model within ground effect maintains a velocity averaging approximately $32 \mathrm{~m} / \mathrm{s}$ after a period of about 30 seconds. On the other hand, the model without a ground effect component is characterized by a waveform of increasing oscillations showing a lack of inherent stability. This waveform is of a relatively lower frequency and with greater amplitude, oscillating at about $20 \mathrm{~m} / \mathrm{s}$. This thus shows that the ground effect has a positive influence on both the performance and stability properties of the proposed model.

\section{Velocity in z-axis}

There are also notable differences seen in the z-direction velocity between simulations of the model with the ground effect influence as compared to the model without this influence. This discrepancy is illustrated in Fig. 14. In the figure, the illustration on the left represents the model influenced by the ground effect, whereas the model on the right shows the model in motion within an unbound air stream. From the illustration, it is clear that the ground effect model exhibits more stability properties as compared to that without the ground effect influence. This is evidenced by a waveform with significantly diminished amplitude for the ground effect model, as compared to the model without ground effect. As oscillations diminish on the left illustration with respect to time, they tend to increase on the right illustration within the same timeframe. Both simulations depict negative values of velocity along the $\mathrm{z}$ direction, which according to the frame of reference points downward. This thus shows a gain in the height of the model, which is in the opposite direction pointing upwards, hence the negative values.

\section{Pitch Rate}

Another element affected by the simulation of the model within the given constraints is the pitch rate. Contrasts between the model with the ground effect influence and that without this influence are illustrated in Fig. 15. In the illustration, the model within the ground effect, on the left, exhibits more stability properties as compared to the model without the ground effect influence, shown on the right. This is evidenced by a waveform whose amplitude diminishes over time, in left illustration, as compared to one where the amplitude is ever-increasing with time, on the right. Pitch rate is affected by the deflection of the elevator control input. After this initial deflection however, the pitch rate reverts to an average of zero with both the presented graphs oscillating around this mark.

\section{Pitch Angle}

The results presented for models also show changes in the pitch angle, which is synonymous with the state denoted by $\mathrm{x} 8$. These changes are illustrated in Fig. 16. In the illustration, while the amplitude for the waveform on the left diminishes over time, the 
waveform on the right has amplitude that increases over time. This thus shows that there is a significant amount of stability exhibited by the model with the ground effect influence as compared to that without this influence.

\section{Z position of Center or Gravity}

The results also reveal changes in the z position of the center of gravity, or the height of the model. These changes are illustrated in Fig. 17. The figure on the left represents z-position or height of the model with the ground effect influences, whereas that on the right represents a model without ground influence parameter. In the first illustration, the height initially increases due to a change in the pitch angle before gradually decreasing. The second illustration illustrates oscillating motion and lack of a steady state, showing the instability of the system without the ground effect influence. The response, shown for the model within the ground effect is typical for an aerodynamic body, since a change in the pitch angle affects lift and therefore the height of the body. It should however be noted, that for an ideal ground- effect vehicle (GEV), the height above the ground surface should be restricted to a few feet.

\section{Angle of Attack}

The results also depict the effect that a change in the angle of attack has on the response of the established dynamic system. Effect on the longitudinal velocity at various pitch angles is used to demonstrate these discrepancies and presented in Fig. 18. The illustration shows velocity in the x-direction at 5 degrees, 17 degrees and 30 degrees angle of attack, respectively. The depicted waveforms show that there are minimal perturbations, synonymous with the least amplitude, at 5 degrees. This translates to minimal vibrations or disturbances of the body at 5 degrees. All the models achieve initial relative stability after about 30 seconds. The model at 17 degrees, however, begins to show signs of instability after about 100 seconds. The model appears to be most stable at five degrees where the velocity is maintained at $30 \mathrm{~m} / \mathrm{s}$. At 17 and 30 degrees pitch angle, some of the initial velocity is lost, with the model settling at about $25 \mathrm{~m} / \mathrm{s}$ for the 17 degrees pitch angle and about $20 \mathrm{~m} / \mathrm{s}$ at 30 degree pitch angle.
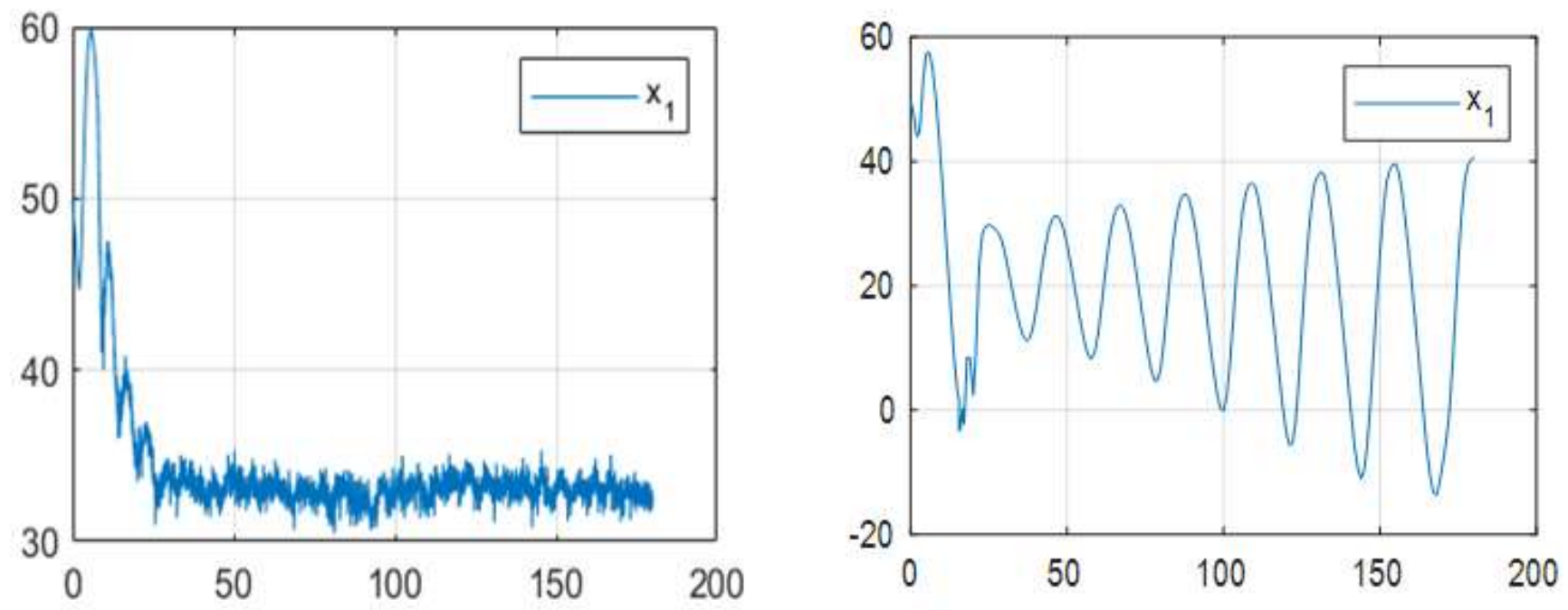

Fig. 12: Comparison of Velocity in X-direction within Ground Effect vs. without Ground Effect
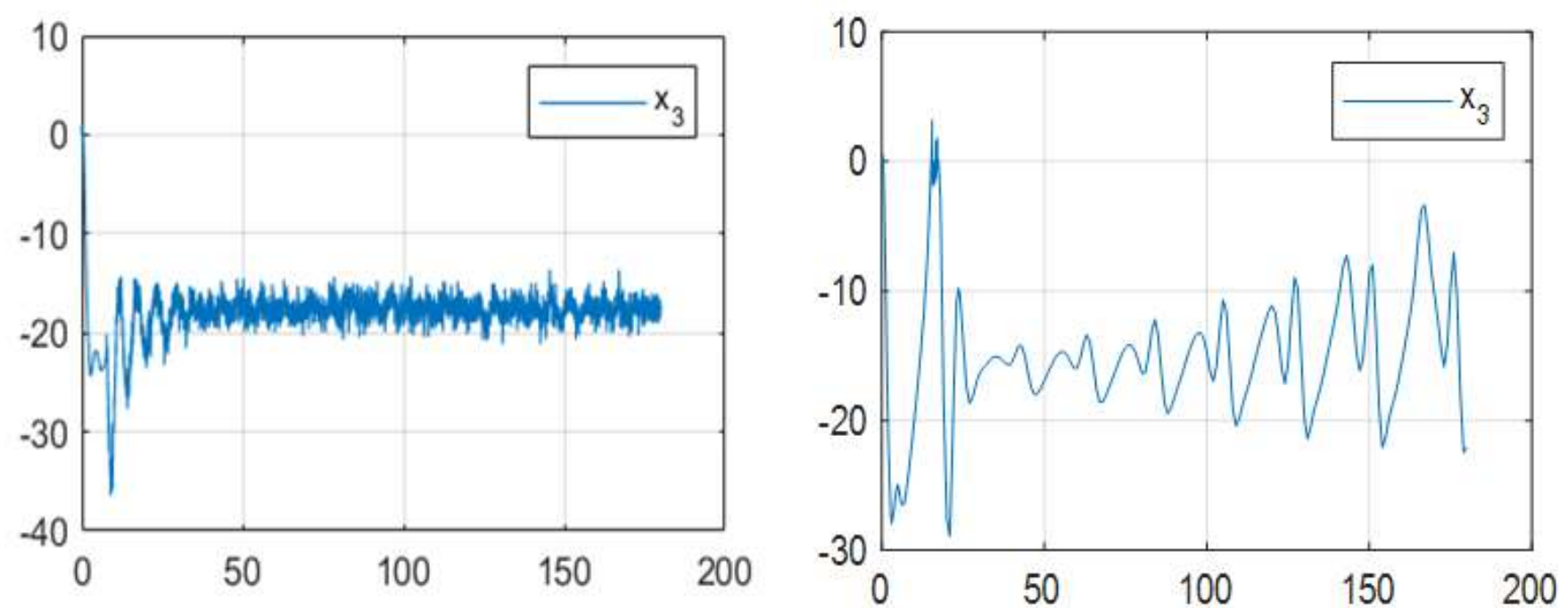

Fig. 13: Comparison of Velocity in z-direction within Ground Effect vs. without Ground Effect 

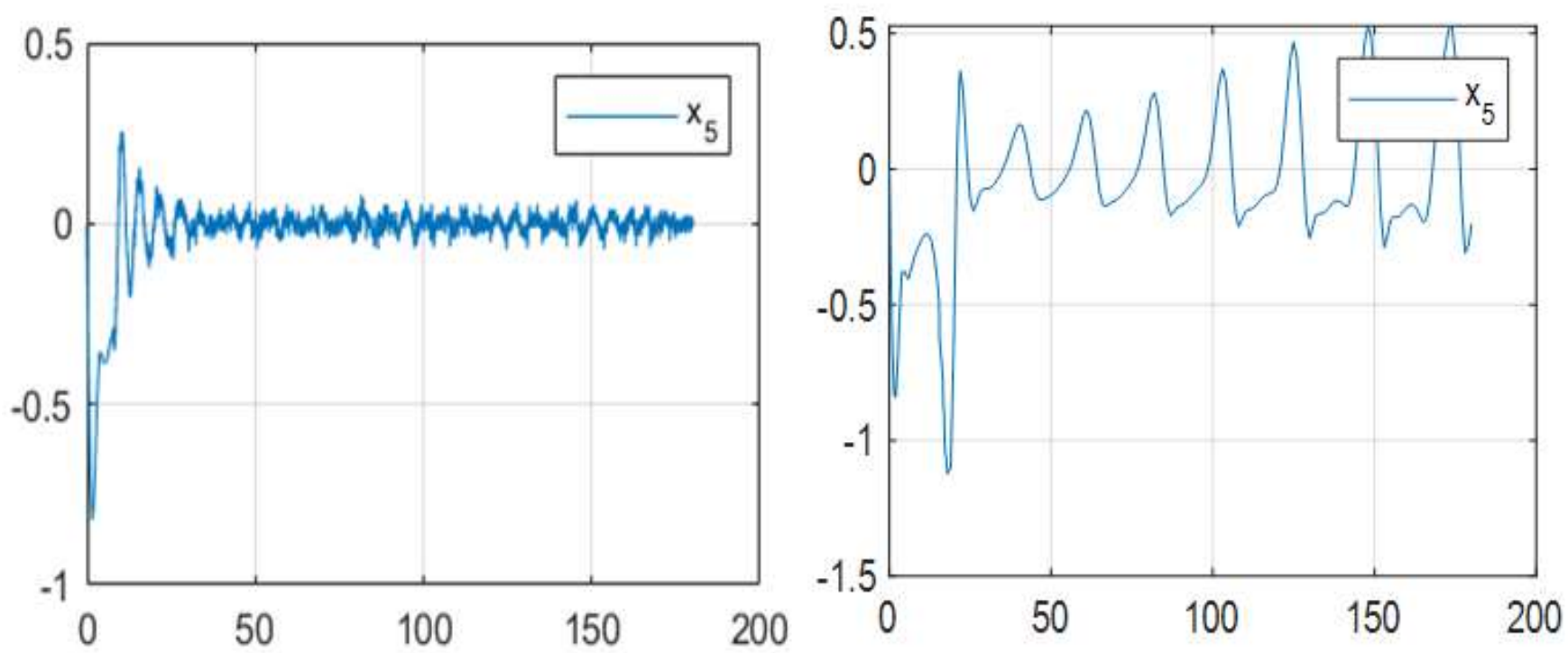

Fig. 14: Comparison of Pitch Angle within Ground Effect vs. without Ground Effect
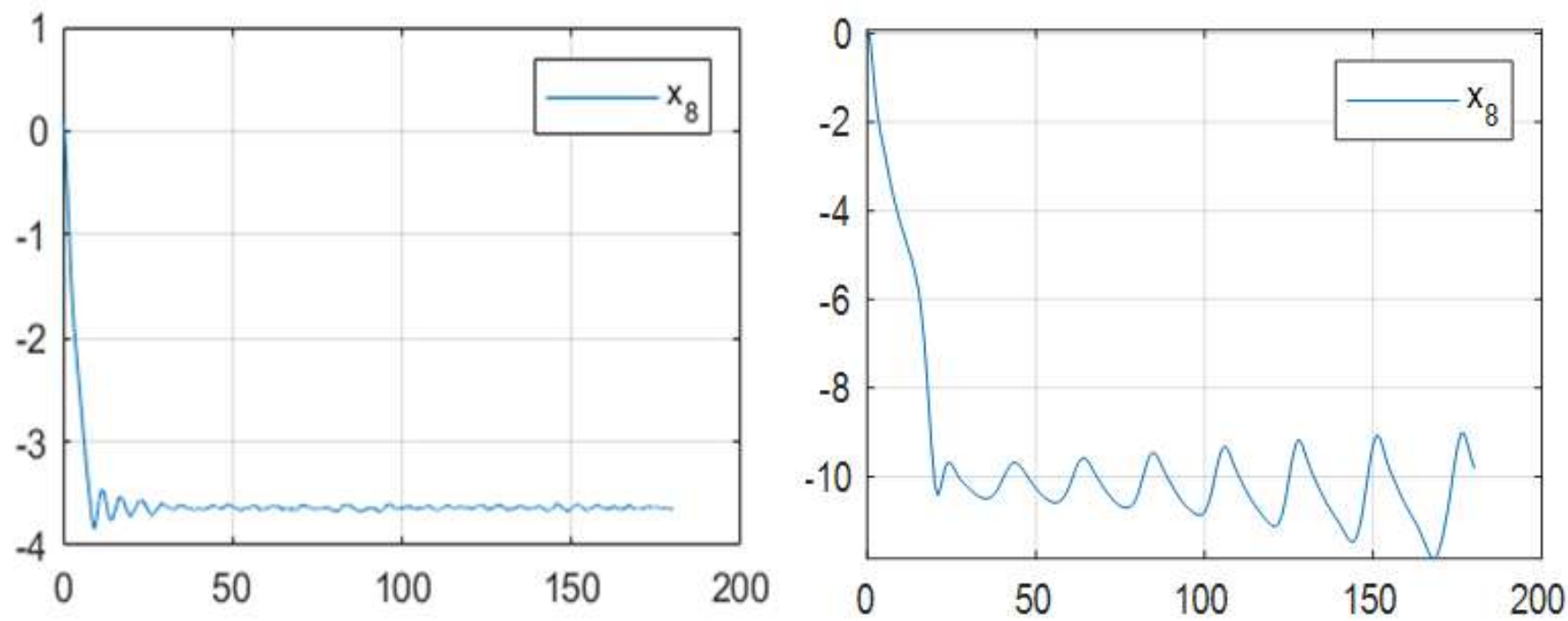

Figure 15. Comparison of Pitch Rate within Ground Effect vs. without Ground Effect
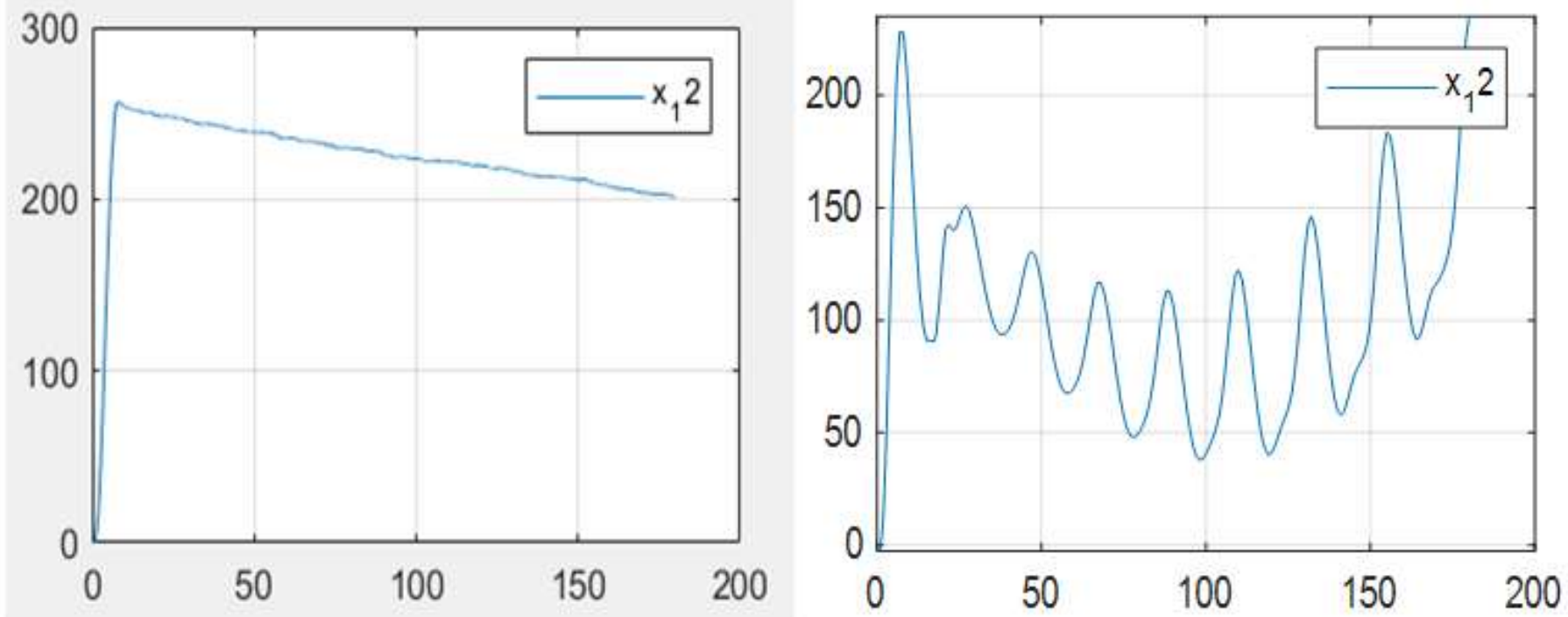

Figure 16. Comparison of Pitch Rate within Ground Effect vs. without Ground Effect 

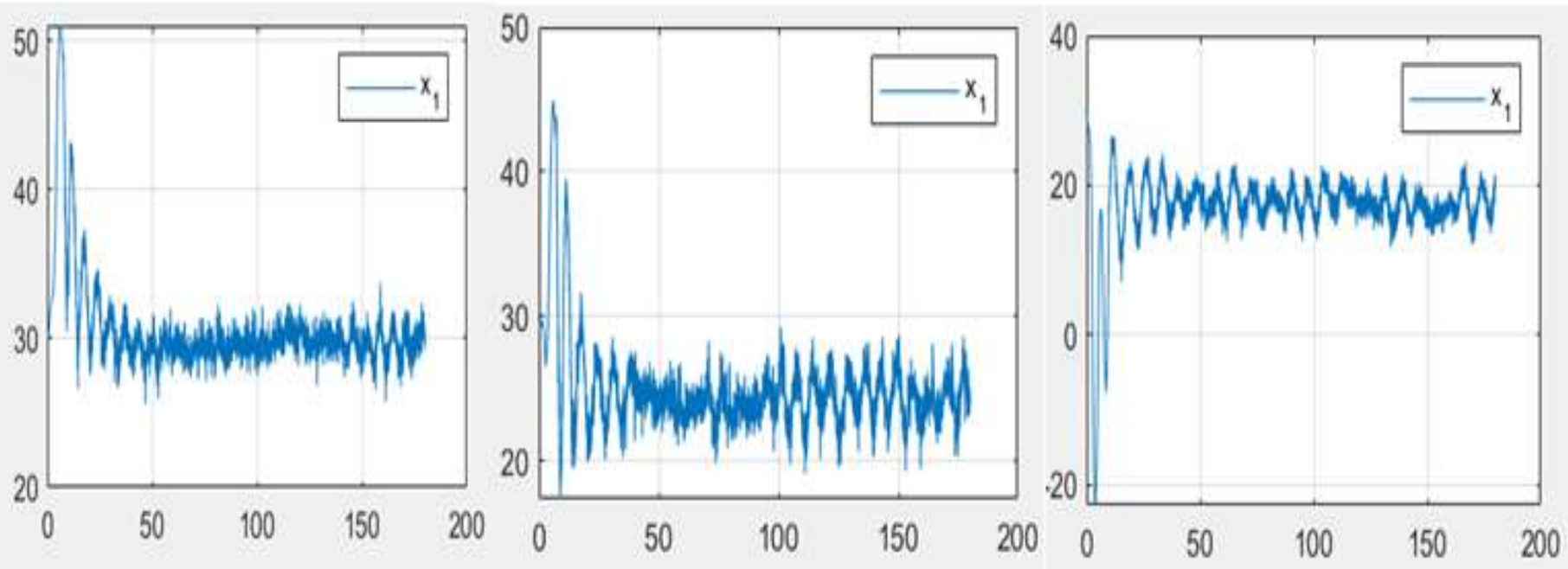

Figure 17. Differences in Longitudinal Velocity at $5^{\circ}, 17^{\circ}$ and $30^{\circ}$ Pitch Angles Respectively

\section{Vertical Motion}

The model is also simulated to portray preliminary results of the concept's dynamics, when vertical thrust is produced by a number of nozzles underneath the FAB. The thrust forces produced by these nozzles are modeled as resultant force acting through the center of gravity in opposition to gravitational force. The resultant force is divided into the combination of force produced by the right set of nozzles fed by Engine fan 1 and the left set of nozzles fed by Engine fan 2. The results are simulated at a throttle setting of 0.13 rads for control inputs $\mathrm{u} 4$ and $\mathrm{u} 5$.

An examination of the velocity along the $\mathrm{x}$-axis as illustrated in figure 1, shows major differences with the model in horizontal motion maintaining a velocity of around $30 \mathrm{~m} / \mathrm{s}$ whereas, this velocity in the vertical mode drops to about $24 \mathrm{~m} / \mathrm{s}$. Results of the horizontal motion also show increased vibrations in comparison to the vertical motion. This can be attributed to increased airflow when the model with movement in the horizontal direction, as compared to vertical motion. These discrepancies are illustrated by Fig. 19.

Differences between the horizontal and vertical mode of the concept's motion also extend to velocity along the z-axis. Although both modes show velocities in the $\mathrm{z}$ direction, the vertical mode of motion has steady and significantly higher velocity along this axis as compared to the horizontal mode. This is because in the horizontal mode, velocity along the z-axis is primarily influenced by the pitch angle and lift as a result of the wing's aerodynamic properties. On the other hand in the vertical mode, velocity in the z-axis is primarily facilitated by the vertical thrust as a result of vectored force from the engines being produced by nozzles in the skirt of the Airboat. Since this force is more consistent, the plots of w exhibit smoother characteristics and thus more stability as shown in Fig. 20.

The final major difference between the horizontal and vertical modes is shown by the z-position in the earth frame, which also denotes the height of the model. In this regard, both the modes depict an increase in height. However, the horizontal mode shows the model's increase in height slowing down after a fraction of the time. On the other hand, z-position of the model in relation to the earth continues to increase consistently throughout the period as shown in Fig. 21. This shows the potential of the vertical mode to sustain a change in the concept's height and thus promote vertical motion.

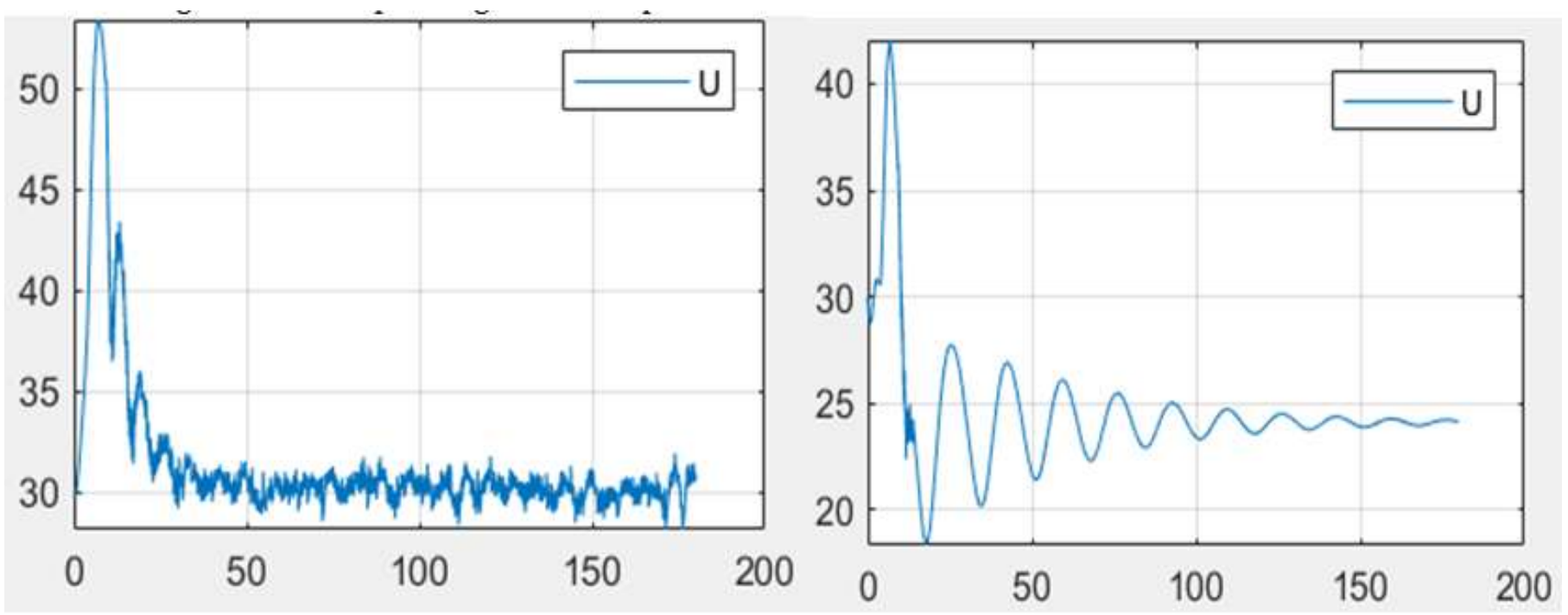

Fig. 18: Velocity in $x$-axis in horizontal motion vs. vertical mode 


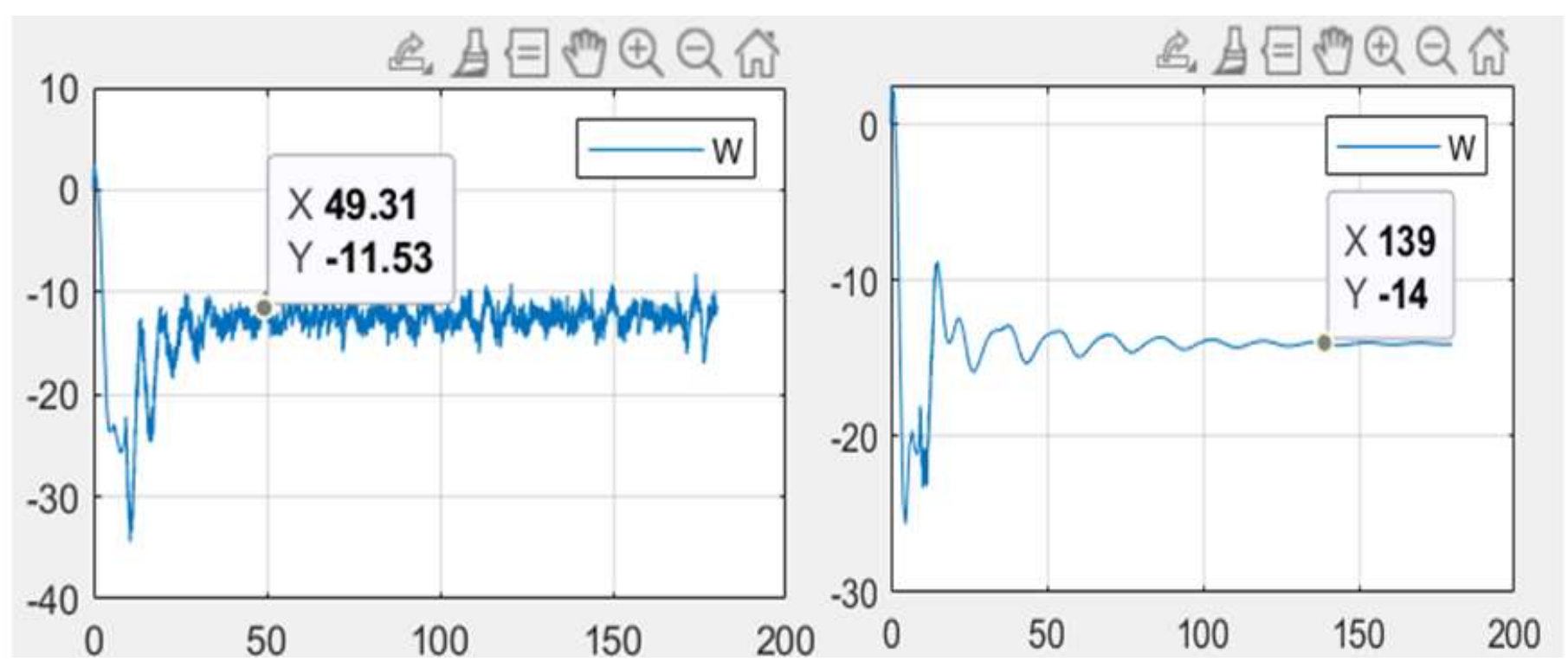

Fig. 19: Velocity in z-axis in horizontal vs. vertical modes
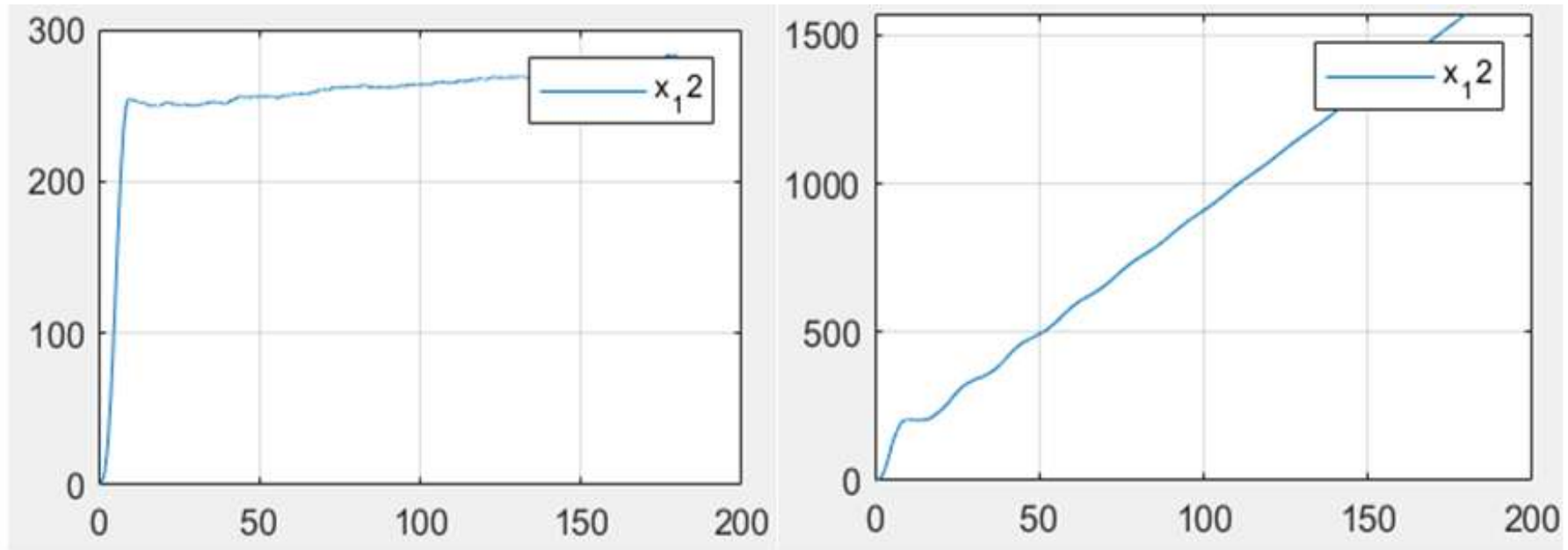

Fig. 20: Change in z-position (height) in horizontal vs. vertical modes

\section{CONCLUSION}

The implementation of the mathematical model for the FAB on the MATLAB platform was useful as a means of determining the controllability of the proposed design. Once implementation of the model had sufficed, the results revealed that indeed, control of the FAB could be achieved with minor changes to the proposed design. Among the changes that were incorporated was the inclusion of a tail section to improve the dynamic properties and stability of the model. Simulation of the design revealed that the incorporation of the ground effect influence parameter was necessary for ideal maneuverability and control of the design. The design also demonstrated that the use vertical motion could be achieved with the vectoring of thrust through nozzles in the skirt section underneath the FAB.

The results presented were based on a non-linear dynamic model. Future works could thus encompass the linearization of the model and the incorporation of a feedback system to minimize the error within the system, as a precursor to achieving a robust control design for the concept. Once robust control has been established, a scaled experimental model could then be developed to test the aerodynamic properties of the concept.

\section{REFERENCES}

[1] S. Jamei, A. Maimun, S. Mansor, N. Azwadi and A. Priyanto, "Design Parametric Study of a Compound Wing-in-Ground Effect. II: Aerodynamics Coefficients", Journal of Aerospace Engineering, vol. 29, no. 1, p. 04015023, 2016. Available: 10.1061/(asce)as.1943-5525.0000497.

[2] Mohd Zaid, M. Z., Arifin, M., “Aerodynamic Design for a High-Speed Airboat Craft”, Universiti Selangor (UNISEL), 2015.

[3] Abramowski, Tomasz. "Numerical investigation of airfoil in ground proximity." Journal of theoretical and applied mechanics vol.45, pp.425-436, 2007.

[4] Wetmore, J. W., and L. I. Turner Jr. "Determination Of Ground Effect From Tests Of A Glider In Towed Flight",1940. 
[5] Rauw, M. “A Simulink Toolbox for Flight Dynamics and Control Analysis.” In Zeist, The Netherlands (Issue February), 2001. Available: http://www.et.byu.edu/ beard/classes/ece682rweb/www-randy/fdcmanual.pdf

[6] Lambrechts, Paul F., Samir Bennani, Gertjan Looye, and Anders Helmersson. "Robust flight control design challenge problem formulation and manual: the research civil aircraft model (RCAM)." Technical Publication TP-088-3, Group for Aeronautical Research and Technology in Europe GARTEUR-FM (AG08), 1997.

[7] "EPPLER 857 AIRFOIL (e857-il)", Airfoiltools.com, 2020. [Online].

Available: http://airfoiltools.com/airfoil/details?airfoil=e857-il. [Accessed: 05- Dec- 2020]. 